\title{
LOWER BOUNDS FOR CORNER-FREE SETS
}

\author{
Ben GREen
}

(Received 24 February, 2021)

\begin{abstract}
A corner is a set of three points in $\mathbf{Z}^{2}$ of the form $(x, y),(x+$ $d, y),(x, y+d)$ with $d \neq 0$. We show that for infinitely many $N$ there is a set $A \subset[N]^{2}$ of size $2^{-(c+o(1)) \sqrt{\log _{2} N}} N^{2}$ not containing any corner, where $c=2 \sqrt{2 \log _{2} \frac{4}{3}} \approx 1.822 \ldots$
\end{abstract}

Let $q, d$ be large positive integers. For each $x \in\left[q^{d}-1\right]$, we may write $\pi(x)=$ $\left(x_{0}, \ldots, x_{d-1}\right) \in \mathbf{Z}^{d}$ for the vector of digits of its base $q$ expansion, thus $x=$ $\sum_{i=0}^{d-1} x_{i} q^{i}$, with $0 \leqslant x_{i}<q$ for all $i$.

For each positive integer $r$, consider the set $A_{r}$ of all pairs $(x, y) \in\left[q^{d}-1\right]^{2}$ for which $\|\pi(x)-\pi(y)\|_{2}^{2}=r$ and $\frac{q}{2} \leqslant x_{i}+y_{i}<\frac{3 q}{2}$ for all $i$.

We claim that $A_{r}$ is free of corners. Suppose that $(x, y),(x+d, y),(x, y+d) \in A_{r}$. Then

$$
\|\pi(x)-\pi(y)\|_{2}^{2}=\|\pi(x+d)-\pi(y)\|_{2}^{2}=\|\pi(x)-\pi(y+d)\|_{2}^{2}=r .
$$

We claim that

$$
\pi(x+d)+\pi(y)=\pi(x)+\pi(y+d) .
$$

To this end, we show that $(x+d)_{i}+y_{i}=x_{i}+(y+d)_{i}$ for $i=0,1, \ldots$ by induction on $i$. A single argument works for both the base case $i=0$ and the inductive step. Suppose that, for some $j \geqslant 0$, we have the statement for $i<j$. Write $x_{\geqslant j}:=$ $\sum_{i \geqslant j} x_{i} q^{i}$, and define $(x+d)_{\geqslant j}, y_{\geqslant j},(y+d)_{\geqslant j}$ similarly. By the inductive hypothesis and the fact that $x+(y+d)=(x+d)+y$, we see that $x_{\geqslant j}+(y+d)_{\geqslant j}=(x+d)_{\geqslant j}+y \geqslant j$. Therefore $x_{j}+(y+d)_{j}=(x+d)_{j}+y_{j}(\bmod q)$. However by assumption we have $\frac{q}{2} \leqslant x_{j}+(y+d)_{j},(x+d)_{j}+y_{j}<\frac{3 q}{2}$, and so $x_{j}+(y+d)_{j}=(x+d)_{j}+y_{j}$. The induction goes through.

With (2) established, let us return to (1). We now see that this statement implies that $\|a\|_{2}^{2}=\|a+b\|_{2}^{2}=\|a-b\|_{2}^{2}=r$, where $a:=\pi(x)-\pi(y)$ and $b:=\pi(x+d)-$ $\pi(x)=\pi(y+d)-\pi(y)$. By the parallelogram law $2\|a\|_{2}^{2}+2\|b\|_{2}^{2}=\|a-b\|_{2}^{2}+\|a+b\|_{2}^{2}$, this immediately implies that $b=0$. Since $\pi$ is injective, it follows that $d=0$ and so indeed $A_{r}$ is corner-free.

The set of all pairs $(x, y)$ with $\frac{q}{2} \leqslant x_{i}+y_{i}<\frac{3 q}{2}$ for all $i$ has size $\left(\frac{3}{4} q^{2}+\right.$ $O(q))^{d}$. Therefore by the pigeonhole principle there is some $r$ such that $\# A_{r} \geqslant$ $\left(d q^{2}\right)^{-1}\left(\frac{3}{4} q^{2}+O(q)\right)^{d}$.

2010 Mathematics Subject Classification Primary 11B25, Secondary 05D10.

The author is supported by a Simons Investigator Grant. 
Now for a given $d$ set $q:=\left\lfloor(2 / \sqrt{3})^{d}\right\rfloor$ and $N:=q^{d}$. Then $A_{r} \subset[N] \times[N], A_{r}$ is free of corners, and

$$
\# A_{r} \geqslant N^{2}\left(d q^{2}\right)^{-1}\left(\frac{3}{4}+O\left(\frac{1}{q}\right)\right)^{d} .
$$

Writing $o(1)$ for a quantity tending to 0 as $N \rightarrow \infty$, we note that $q=\left(\frac{2}{\sqrt{3}}+o(1)\right)^{d}$ and that $d=(1+o(1)) \sqrt{\frac{\log _{2} N}{\log _{2}(2 / \sqrt{3})}}$. A short calculation then confirms that

$$
\# A_{r} \geqslant N^{2} 2^{-(c+o(1)) \sqrt{\log _{2} N}},
$$

where $c=2 \sqrt{2 \log _{2} \frac{4}{3}} \approx 1.822 \ldots$

Remark. The construction came about by a careful study of the recent preprint of Linial and Shraibman [1], where they used ideas from communication complexity to obtain a bound with $c=2 \sqrt{\log _{2} e} \approx 2.402 \ldots$, improving on the previously best known bound with $c=2 \sqrt{2} \approx 2.828 \ldots$ which comes from Behrend's construction. By bypassing the language of communication complexity one may simplify the construction, in particular avoiding the use of entropy methods. This yields a superior bound.

\section{References}

[1] N. Linial and A. Shraibman, Larger corner-free sets from better NOF exactly- $N$ protocols, preprint, arxiv:2102.00421.

Ben Green

Mathematical Institute,

Radcliffe Observatory Quarter,

Woodstock Road,

Oxford OX2 6GG,

England

ben.green@maths.ox.ac.uk 